\title{
Effect of Magnetic Susceptibility Gradient on the Magnetomigration of Rare-Earth Ions
}

\author{
Isadora R. Rodrigues, ${ }^{*, \dagger}$ Liubov Lukina, ${ }^{\dagger}$ Sam Dehaeck, ${ }^{\ddagger}$ Pierre Colinet, ${ }^{\ddagger}$ \\ Koen Binnemans, ${ }^{\top}$ and Jan Fransaer ${ }^{\dagger}$ \\ $\dagger$ Department of Materials Engineering, KU Leuven, Kasteelpark Arenberg 44, box 2450, \\ B-3001 Heverlee, Belgium \\ $\ddagger$ Université Libre de Bruxelles (ULB), TIPs (Transfers, Interfaces and Processes), CP \\ 165/67, Av. F. D. Roosevelt 50, 1050 Brussels, Belgium \\ \Department of Chemistry, KU Leuven, Celestijnenlaan 200F, box 2404, B-3001 Heverlee, \\ Belgium \\ E-mail: isadora.reisrodrigues@kuleuven.be \\ Phone: +32 16 373677. Fax: + 3216321990
}

The Journal of Physical Chemistry C, 2019, 123 (37), 23131-23139

https://doi.org/10.1021/acs.jpcc.9b06706 


\begin{abstract}
Magnetomigration of rare-earth ions activated by thermal and evaporation-based gradients was demonstrated with the help of Mach-Zehnder interferometry. Magnetic susceptibility gradients were induced in aqueous solutions of rare-earth ions by local heating/cooling or by evaporation of the solvent. Both methods yielded the enrichment of strongly paramagnetic $\mathrm{Dy}^{3+}$ ions in the region of the highest magnetic field. Three different orientations of the magnetic field were tested using temperature as the source of magnetic susceptibility gradient. Enhanced magnetomigration was observed when gradients of magnetic field and magnetic susceptibility were non-collinear, indicating that the rotational component of the magnetic force drives the process. Additionally, four rare-earth ions with distinct values of magnetic susceptibility were studied: the diamagnetic ion $\mathrm{Y}^{3+}$, and the paramagnetic ions $\mathrm{Nd}^{3+}, \mathrm{Gd}^{3+}$ and $\mathrm{Dy}^{3+}$. A strong correlation between the obtained magnetomigration and the magnetic susceptibility of the rare-earth ions was found. When heating/cooling or evaporation were stopped during magnetization experiments, the magnetic effect gradually faded. This demonstrates that the presence of magnetic susceptibility gradients in the system is crucial for the magnetomigration. These findings are of importance for the development of a magnetic separation process for rare-earth ions.
\end{abstract}

\title{
Keywords
}

Lanthanides; magnetic fields; magnetic separation; paramagnetism; rare-earths. 


\section{Introduction}

Due to the strong chemical similarity of the rare-earth (RE) ions, industrial separation methods based on liquid-liquid extraction require a large number of separation steps. ${ }^{1}$ On the other hand, physical properties such as magnetic moment $(\mu)$ and magnetic susceptibility $(\chi)$ vary substantially from one trivalent $\mathrm{RE}^{3+}$ ion to another. Values of $\chi$ range from negative for the diamagnetic ions to positive for the paramagnetic ions. ${ }^{2}$ Droplets of concentrated $\mathrm{RE}^{3+}$ solutions were shown to develop different velocities proportional to the magnitude of $\chi$ when subjected to magnetic fields. ${ }^{3}$ Hence, separation methods based on magnetic properties are particularly relevant for RE compounds. Magnetic separation of macroscale objects is a well-known method used in ore processing, food industry and biomedical diagnostics. In contrast, when downscaling from particles or droplets to much smaller molecules and ions, the situation becomes more complicated. ${ }^{4-6}$ In a closed system containing a homogeneous solution exposed to a magnetic field at room temperature, the kinetic energy of ions is about five orders of magnitude greater than the magnetic energy, even for the strongly paramagnetic $\mathrm{Dy}^{3+}$ ion. ${ }^{7-9}$ Despite this fact, several studies on the magnetic mobility of ions have been reported in the literature.

In the 1950s, Noddack et al. were the first researchers to demonstrate the potential use of magnetic fields to separate solutions of RE salts in fractions of different magnetic susceptibilities. ${ }^{10-12}$ In the $1980 \mathrm{~s}$, Mukherjee et al. obtained separation of several binary mixtures of para- and diamagnetic RE ions using magneto-paper electrophoresis. ${ }^{13}$ Later, reports mainly addressed magnetomigration, i.e. movement of paramagnetic ions of one type under the influence of a magnetic field, rather than magnetic separation. ${ }^{14,15}$ Fujiwara et al. demonstrated the motion of transition metal ions in solutions spotted on a silica gel support in the presence of magnetic fields. ${ }^{16-19}$ Mach-Zehnder interferometry (MZI) was used to observe in situ the migration of $\mathrm{Mn}^{2+}$ and $\mathrm{Gd}^{3+}$ paramagnetic ions exposed to

magnetic fields. ${ }^{20,21}$ A permanent magnet was placed on top of a cell containing the solution and a concentration increase was observed in the upper part of the cell. Pulko et 
al. performed similar experiments using strongly paramagnetic $\mathrm{Dy}^{3+}$ ions. $^{22}$ In our initial work on magnetomigration of RE ions, where a homogeneous $\mathrm{Dy}^{3+}$ solution was exposed to a magnetic field, no migration of ions was detected. ${ }^{8}$ In contrast, when a magnetic susceptibility gradient $(\nabla \chi)$ was introduced to the system by solvent evaporation, magnetomigration was observed. The same phenomenon was investigated by Lei et al. ${ }^{23}$

In this paper it is shown that different approaches of introducing $\nabla \chi$ to a solution of paramagnetic ions can induce magnetomigration. Experiments were performed with thermal and evaporation-based gradients leading to the enrichment of paramagnetic ions in the presence of an external magnetic field. The contribution of the rotational component of the magnetic force to the magnetomigration was highlighted in experiments with thermal gradient and three different orientations of the magnetic field. To study the impact of distinct values of molar magnetic susceptibility on the magnetomigration process, four different $\mathrm{RE}$ ions were used with evaporation-based gradient: $\mathrm{Y}^{3+}, \mathrm{Nd}^{3+}, \mathrm{Gd}^{3+}$ and $\mathrm{Dy}^{3+}$. Additionally, to emphasize the role of $\nabla \chi$ in the magnetomigration, the effect of discontinuing the heating/cooling and the evaporation during magnetization was investigated.

\section{Theoretical Background}

When a solution is magnetized in an external field, it acquires a magnetic energy $E_{\text {mag }}$ $\left[\mathrm{J} \mathrm{m}^{-3}\right]$ which is expressed as: ${ }^{3,7,8,24-26}$

$$
E_{\mathrm{mag}}=-\frac{1}{2} M B
$$

where $M$ is the magnetization $\left[\mathrm{A} \mathrm{m}^{-1}\right]$, and $B$ is the magnetic field flux $[\mathrm{T}]$. In cases where $M=\frac{\chi_{\mathrm{sol}} B}{\mu_{0}}$ (e.g. diamagnetic and paramagnetic solutions), Eq. 1 can be written as:

$$
E_{\mathrm{mag}}=-\frac{1}{2 \mu_{0}} \chi_{\mathrm{sol}} B^{2}
$$


where $\mu_{0}=4 \pi \times 10^{-7} \mathrm{~T} \mathrm{~m} \mathrm{~A}^{-1}$ is the magnetic permeability of the vacuum; and $\chi_{\text {sol }}$ $[-]$ is the volumetric magnetic susceptibility of the solution. The negative sign accounts for the fact that paramagnetic species lower their energy when placed in a magnetic field while diamagnetic species increase their energy. Hence, for paramagnetic solutions $\chi_{\text {sol }}>0$, therefore $E_{\mathrm{mag}}<0$. On the other hand, for a diamagnetic solutions $\chi_{\text {sol }}<0$, and $E_{\text {mag }}>$ 0. $\chi_{\text {sol }}$ is related to the molar magnetic susceptibility $\chi_{\mathrm{m}}\left[\mathrm{m}^{3} \mathrm{~mol}^{-1}\right]$ and concentration $c$ $\left[\mathrm{mol} \mathrm{m}^{-3}\right]$ of species $i$ in solution:

$$
\chi_{\mathrm{sol}}=\sum_{i} \chi_{\mathrm{m} i} c_{i}
$$

According to Curie's law, $\chi_{\mathrm{m}}$ is temperature-dependent: ${ }^{7}$

$$
\chi_{\mathrm{m}}=\frac{C_{\mathrm{m}}}{T}
$$

where $\mathrm{C}_{\mathrm{m}}$ is the Curie constant $\left[\mathrm{m}^{3} \mathrm{~mol}^{-1} \mathrm{~K}\right]$ and $T$ is the temperature $[\mathrm{K}]$ :

$$
\mathrm{C}_{\mathrm{m}}=\frac{\mu_{0} \mathrm{~N}_{\mathrm{A}} g^{2} \mu_{\mathrm{B}}^{2} S(S+1)}{3 \mathrm{k}_{\mathrm{B}}}
$$

where $\mathrm{N}_{\mathrm{A}}=6.022 \times 10^{-23} \mathrm{~mol}^{-1}$ is Avogadro's number; $\mu_{\mathrm{B}}=9.274 \times 10^{-24} \mathrm{~A} \mathrm{~m}^{2}$ is the Bohr magneton; $\mathrm{g}=2.0023$ is the Landé g-factor; $S$ is the electronic spin quantum number of species $i$; and $\mathrm{k}_{\mathrm{B}}=1.38 \times 10^{-23} \mathrm{~J} \mathrm{~K}^{-1}$ is the Boltzmann constant (Eq. 5 holds for spin-only species).

The magnetic volumetric force acting on a solution in an external magnetic field can be obtained by taking the first derivative of the magnetic energy $F_{\mathrm{mag}}=-\nabla E_{\mathrm{mag}}\left[\mathrm{N} \mathrm{m}^{-3}\right]$ :

$$
F_{\mathrm{mag}}=F_{\nabla \chi}+F_{\nabla B}=\frac{1}{2 \mu_{0}} \nabla \chi_{\mathrm{sol}} B^{2}+\frac{1}{2 \mu_{0}} \chi_{\mathrm{sol}} \nabla B^{2}
$$

This derivation assumes that $\chi_{\text {sol }}$ is independent of the magnitude of the magnetic field, which is valid for diamagnetic and paramagnetic solutions. ${ }^{7}$ The first term in the magnetic 
force is the magnetic susceptibility gradient force $F_{\nabla \chi}$, resulting from a uniform magnetic field acting on a solution with a non-uniform magnetic susceptibility $\left(\nabla \chi_{\text {sol }}\right)$, with typical value of $10^{4} \mathrm{~N} \mathrm{~m}^{-3}$ (for e.g. $B=1 \mathrm{~T}$ acting on a paramagnetic solution of $c=1 \mathrm{~mol} \mathrm{dm}^{-3}$ and $\chi_{\mathrm{m}}$ $\left.=10^{-8} \mathrm{~m}^{3} \mathrm{~mol}^{-1}\right) \cdot{ }^{25}$ The second term of the magnetic force is the magnetic field gradient force $F_{\nabla B}$. This force appears when a non-uniform magnetic field is acting on a solution with uniform magnetic susceptibility, and has magnitude of $10^{1} \mathrm{~N} \mathrm{~m}^{-3}$ (for similar conditions as described above). ${ }^{25}$ Despite the fact that $F_{\nabla \chi}$ is larger than $F_{\nabla B}$, neither of these forces can exert any significant influence on ions in a solution since they are overpowered by a coexisting diffusion volumetric force $F_{\mathrm{D}}=\mathrm{R} T \nabla c$, which has the magnitude of $10^{10} \mathrm{~N} \mathrm{~m}^{-3}$ (for $\mathrm{R}=$ $8.314 \mathrm{~J} \mathrm{~K}^{-1} \mathrm{~mol}^{-1}$ the gas constant and assuming $\left.T=298 \mathrm{~K}\right) .{ }^{25,27}$ Therefore, cases of magnetomigration observed in experiments with non-uniform magnetic susceptibility, which were previously attributed to $F_{\nabla \chi},{ }^{8}$ are actually due to the contribution of the rotational component of the magnetic field gradient force $\nabla \times F_{\nabla \mathrm{B}}$ acting on the regions of the solution with non-vanishing $\nabla \chi$ and $\nabla B$. Taking into acount that $F_{\nabla B}$ is a non-conservative force when $\nabla \chi \neq 0, \nabla \times F_{\nabla \mathrm{B}}$ is expressed by: ${ }^{28-30}$

$$
\nabla \times F_{\nabla \mathrm{B}}=\frac{1}{2 \mu_{0}} \nabla \chi_{\text {sol }} \times \nabla B^{2}
$$

The derivation of the rotational component of $F_{\nabla B}$ is shown in Section 1 of the Supporting InformationAs can be seen in Eq. 7, the rotational component of the magnetic force only exists when these conditions are met: the presence of $\nabla B^{2}$ and $\nabla \chi_{\text {sol }}$ in the system; and a non-collinearity of these two gradients. ${ }^{27}$ To summarize, the magnetomigration in external magnetic fields proceeds as a magnetically induced motion inside paramagnetic solutions resulting in local ionic enrichment due to the activation of $\nabla \times F_{\nabla \mathrm{B}} .{ }^{8,20,21,23}$

In experiments with thermal gradients demonstrated in this paper, regions of $\nabla \chi_{\text {sol }}$ are created in the solution due to the temperature dependence of $\chi_{\mathrm{m}}$ (Eq. 4). Additionally, minor concentration gradients are generated due to Soret effect. ${ }^{31}$ In the case of evaporation-based gradient, the local increase in the ionic concentration introduces concentration gradients to 
the system, thus creating regions of $\nabla \chi_{\text {sol }}$ (Eq. 3). Slight effects on $\chi_{\mathrm{m}}$ are also present due to the evaporative cooling of the solvent.

\section{Experimental}

Four $\mathrm{RE}$ ions $\left(\mathrm{Y}^{3+}, \mathrm{Nd}^{3+}, \mathrm{Gd}^{3+}\right.$ and $\left.\mathrm{Dy}^{3+}\right)$ were used in the form of nitrate salts $\mathrm{RE}\left(\mathrm{NO}_{3}\right)_{3} \cdot x \mathrm{H}_{2} \mathrm{O}$ (where $x=5$ or 6 ) of $99.9 \%$ purity (Alfa Aesar). Solutions of $1.0 \mathrm{~mol}$ $\mathrm{dm}^{-3}$ concentration were prepared by dissolving the corresponding metal salt in deionized water. To avoid the hydrolysis of the cations, the $\mathrm{pH}$ was adjusted to 3.0 by adding a few drops of concentrated $\mathrm{HNO}_{3}$. The concentrations were determined by ICP-OES (Varian $720 \mathrm{ES}$ ) after dilutions to $10 \mathrm{ppm}$. The measurements had a relative standard deviation of $\pm 3 \%$ for triplicates of the same measurement. Changes in the refractive index of the solutions were monitored in situ using Mach-Zehnder interferometry (MZI). ${ }^{20-22}$ The experimental description of MZI is given in Section 2 in the Supporting Information. Using a suitable demodulation technique based on the Fourier transform method, refractive indices were computed across the cell containing the RE solution for each fringe pattern. ${ }^{32,33}$ With MZI, it is possible to determine the dimensionless refractive index with a resolution of $10^{-6} .{ }^{33}$ Based on the refractive index values, iso-concentration contour plots or MZI profiles are obtained. All the refractive indices were averaged for the full length along the full height of the cell. The refractive indices of 0.0 to $1.0 \mathrm{~mol} \mathrm{dm}{ }^{-3} \mathrm{Dy}^{3+}$ solutions were measured using a refractometer (Anton Paar Abbemat 200). From the linear relation between the refractive index and the concentration (Figure S4 in the Supporting Information), concentration changes during experiments were calculated. Experiments were performed in a thermostated room at $\left(21^{\circ} \mathrm{C} \pm 1{ }^{\circ} \mathrm{C}\right)$ using UV quartz cells (Hellma): 117.100F (screw cap closed cell, 3.5 mL) and 100.10.20.100OS (open cell, $3.5 \mathrm{~mL}$ ). The

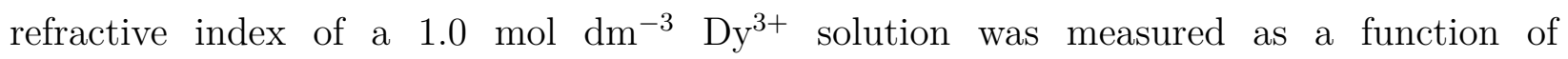
temperature (Figure S5 in the Supporting Information). The magnetic field sources were a 
cuboid Nd-Fe-B magnet $50 \times 50 \times 25 \mathrm{~mm}$, grade N40, and a disc Nd-Fe-B magnet $20 \times 20$ mm, grade N42 (Supermagnete). The strength of the magnetic field was measured with a Gauss meter (475 Lake Shore Cryotronics) by moving the probe away from the central point of the magnet surface in steps of $1 \mathrm{~mm}$ (Figure $\mathrm{S} 6$ in the Supporting Information). The volumetric magnetic susceptibilities of the $\mathrm{RE}^{3+}$ solutions were measured with an Evans balance (MSB Auto, Sherwood Scientific Ltd). The temperature dependence of the magnetic susceptibility was measured for a $1.0 \mathrm{~mol} \mathrm{dm}^{-3} \mathrm{Dy}^{3+}$ solution (Figure $\mathrm{S} 7$ in the Supporting Information). The average deviation of the magnetic susceptibility measurements was $\pm 0.25 \%$ for triplicates of the same solution. The change in the

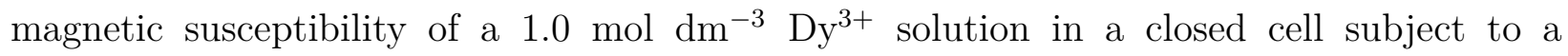
temperature gradient was simulated using the finite element software Comsol 5.2 (Section 4 in the Supporting Information).

\section{Magnetomigration with a thermal gradient}

A heating/cooling device was designed to introduce temperature differences $(\Delta T)$ to the RE solutions inside a closed cell. The device consists of two Peltier elements $(37.9 \mathrm{~W}$, 3.9 A, $15.7 \mathrm{~V}, 30 \times 30 \mathrm{~mm}$, Adaptive) connected to a power supply to provide controlled heating on top of the cell and controlled cooling on the bottom. The cell used in the first configuration was a 100.10.20.100OS (Hellma), reduced in size to $10 \mathrm{~mm} \times 10 \mathrm{~mm} \times 10$ $\mathrm{mm}$. For experiments with magnetization, after 5 minutes of heating/cooling, a magnet was placed in the vicinity of the device. The magnet was kept there for the duration of the magnetization process. The magnetic field was applied in three different orientations related to $\nabla \chi$ : parallel, at the bottom of the cell (Figure 1a); parallel, at the top of the cell (Figure 1b); and perpendicular, at the side of the cell (Figure 1c). 

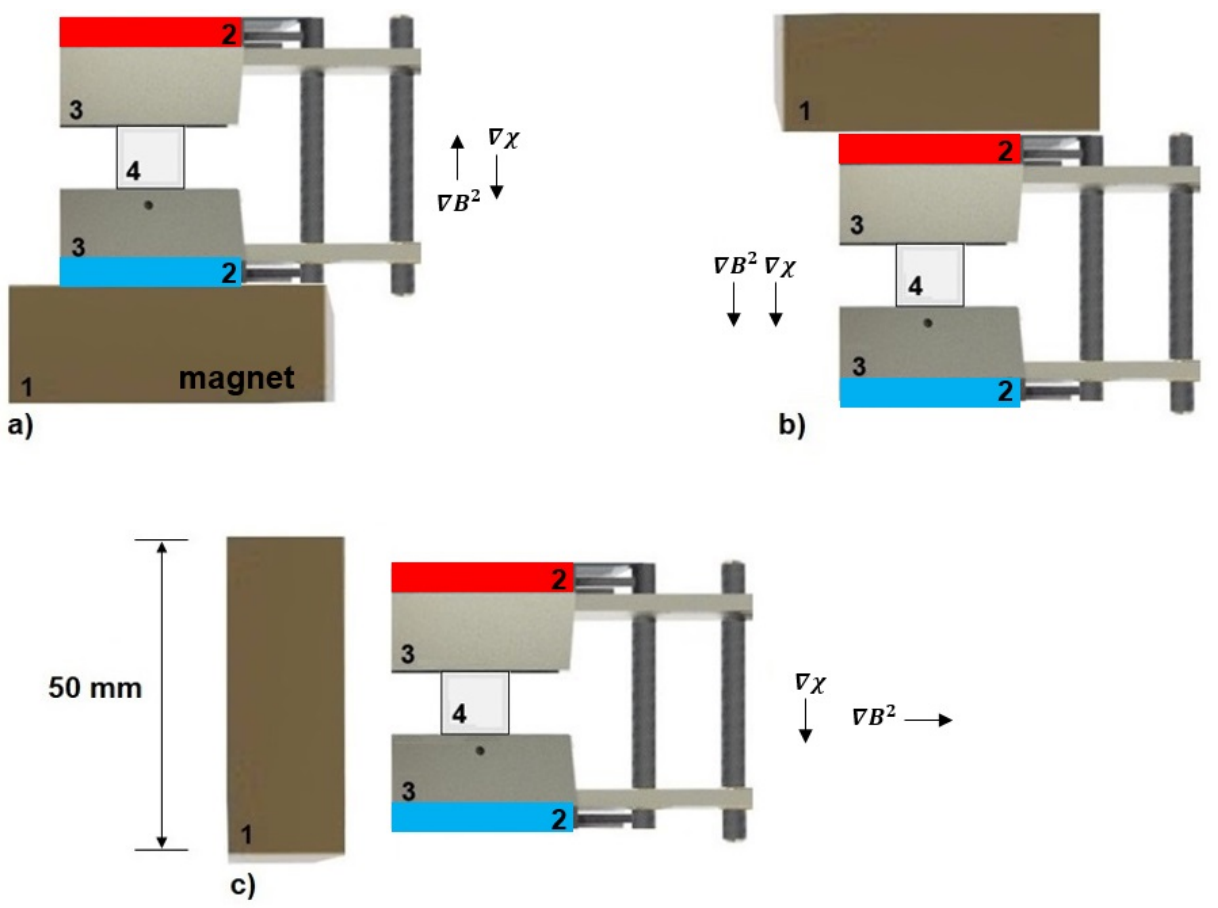

Figure 1: Scheme of configuration 1 of the heating/cooling device with the magnet positioned a) at the bottom, b) at the top and c) at the side of the cell; where 1) Nd-Fe-B magnet; 2) two Peltier elements connected to a power supply; 3) two stainless steel blocks connecting cell walls to the Peltier elements; and 4) closed cell containing a RE solution.

A second configuration of the heating/cooling device was tested, where heating and cooling were applied from the sides of a 100.10.20.100OS cell (Hellma). After 5 minutes of heating/cooling, the magnet was placed $1 \mathrm{~mm}$ from the top of the cell (Figure 2). The magnet was kept there for the duration of the magnetization process and removed for the demagnetization step. This configuration allowed for a qualitative observation of the processes occurring during magnetization and demagnetization. Based on the calibration of the Peltier elements (Figure S10 in the Supporting Information), currents of \pm 0.2 A were selected for the experiments, which resulted in an increase and decrease of $5{ }^{\circ} \mathrm{C}$ from ambient temperature. 


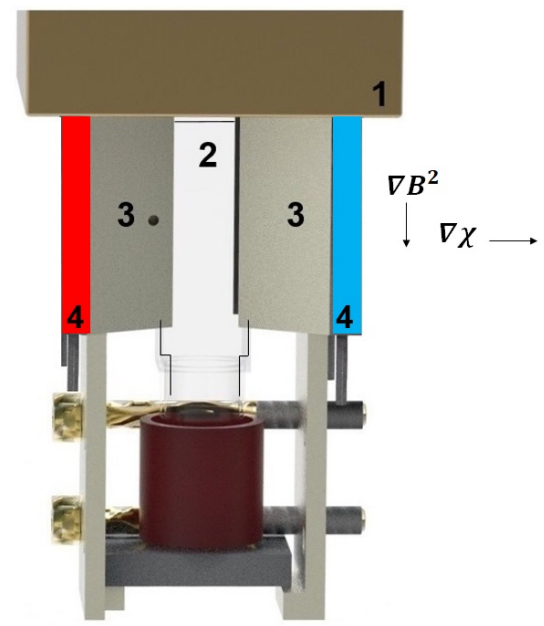

Figure 2: Scheme of configuration 2 of the heating/cooling device: 1) Nd-Fe-B magnet; 2) closed cell containing a RE solution; 3) two stainless steel blocks connecting cell walls to the Peltier elements; and 4) two Peltier elements connected to a power supply.

\section{Magnetomigration with an evaporation-based gradient}

The glass bottom of a 100.10.20.100OS cell (Hellma) was replaced with a PTFE membrane (ThermoFisher Scientific) in order to allow evaporation of water. In the beginning of an experiment, the magnet was positioned $1 \mathrm{~mm}$ from the bottom of the cell and kept there during the magnetization step. For the tests without magnetization, a square block of stainless steel with the same dimensions as the magnet was used while all other experimental parameters were kept the same, ensuring similar evaporation rates for both experiments (Figure 3). The evaporation rate was determined by monitoring the mass of the cell for 50 minutes (Figure S11 in the Supporting Information). 


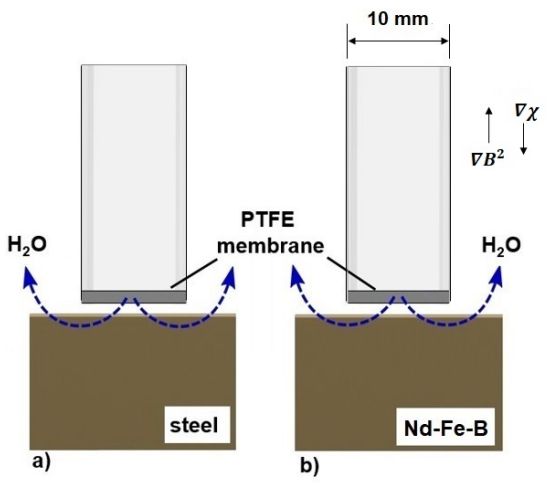

Figure 3: Schematic of the setup used during magnetization experiments with an evaporation-based concentration gradient. The cell is closed on the top and covered with a PTFE membrane at the bottom: a) evaporation experiment without magnet; b) magnetomigration experiment with evaporation-based gradient.

\section{Effect of a non-static magnetic field on the magnetomigration}

A non-static magnetic field was applied during experiments of magnetomigration with an evaporation-based gradient. The responsive behavior of enriched layers of a paramagnetic solution formed at the bottom of the cell was tested. A field concentrator made of grainoriented electrical steel (ArcelorMittal) was mounted on the disc magnet (Figure 4). The magnet was moved from side to side along the bottom of the cell in a velocity of $5 \mathrm{~mm} \mathrm{~s}^{-1}$ with 10 seconds of rest at each position. 

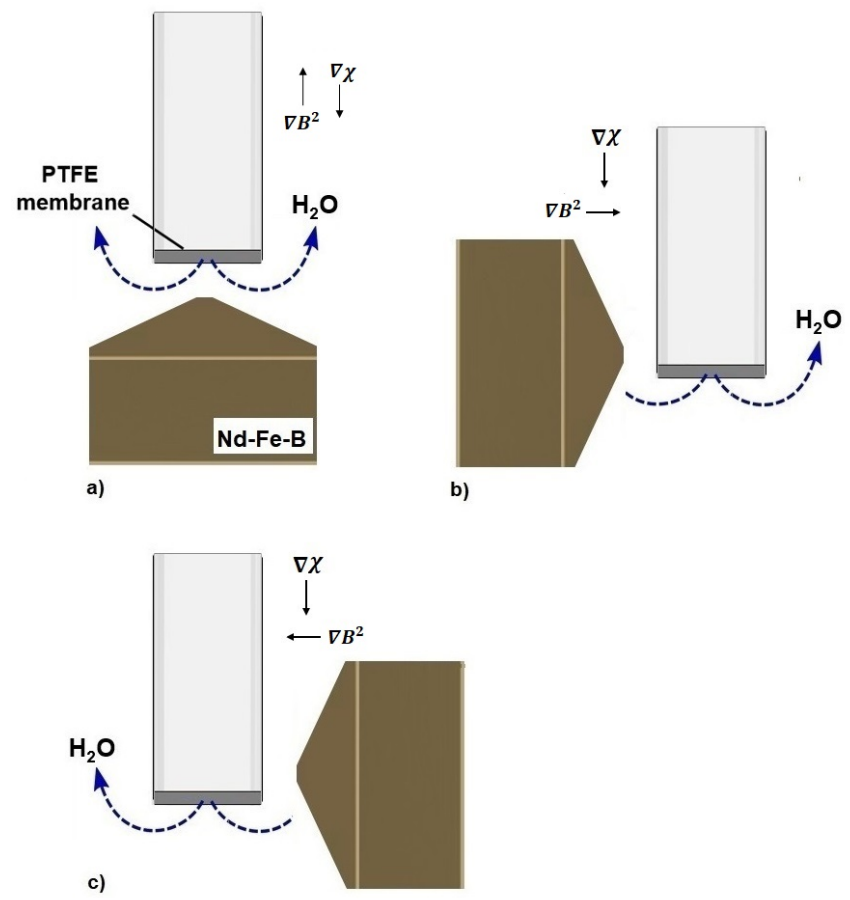

Figure 4: Schematic of the setup used during magnetization experiments with an evaporation-based concentration gradient and a non-static magnetic field. The magnet moved: a) from the center; b) to the left side; and c) to the right side of the cell.

\section{Magnetomigration experiments with removal of thermal and evaporation-based gradients}

These experiments were conducted as described in the previous sections (for thermal and evaporation-based gradients); however, the external sources of $\nabla \chi$ were removed during magnetization. For the experiments with a thermal gradient, the current on the Peltier elements was turned off and $\Delta T$ gradually reduced to zero. For the experiments with an evaporation-based gradient, after a few minutes of magnetization, a thin sheet of ethylenevinyl acetate (EVA) foam was positioned inside the gap between the magnet and the cell, thus preventing further evaporation. The EVA foam had no impact on the strength of the magnetic field. 


\section{Results and Discussion}

\section{Magnetomigration with a thermal gradient}

When a thermal gradient is applied to a paramagnetic solution, regions of $\nabla \chi$ form according to Curie's law (Eq. 4). In the presence of an external magnetic field, these gradients are expected to activate the rotational component of the magnetic field gradient force, inducing the magnetomigration of paramagnetic species. ${ }^{8,23}$ To test this assumption with the help of MZI, experiments were performed in a $1.0 \mathrm{~mol} \mathrm{dm}^{-3} \mathrm{Dy}^{3+}$ solution. Stable conditions for the experiments in absence of natural convection were obtained using configuration 1 of the heating/cooling device. Figure 5a shows the MZI profile for an experiment where the solution was subjected to a thermal gradient operating alone, $\Delta T=$ $10{ }^{\circ} \mathrm{C}$. The temperature gradient due to $\Delta T$ resulted in a linear profile inside the cell. In the next experiment, the thermal gradient was coupled with an external magnetic field applied from the bottom of the cell. Figure 5b shows the profile developed during 30 minutes of magnetization. Here, the effect of the magnetic enrichment on the solution was overpowered by the stratification due to $\Delta T$. When the magnetic field was applied from the top of the cell for 30 minutes, a slight increase in the contraction of the profile was observed, attributed to the magnetomigration of $\mathrm{Dy}^{3+}$ ions towards the higher magnetic field (Figure 5c). As discussed in the theoretical section, the necessary condition for the rotational component of the magnetic field gradient force to act on a solution is the

non-collinearity of the gradients of magnetic field and magnetic susceptibility (Eq.7). ${ }^{28,30,34}$ Even though curved magnetic field lines are distributed in the space around the magnet (Figure S6 in the Supporting Information), positioning the magnet parallel to the Peltier elements results in a high degree of collinearity in the system. This explains the small magnetic effect obtained with the magnetic field applied from the bottom and the top of the cell. 


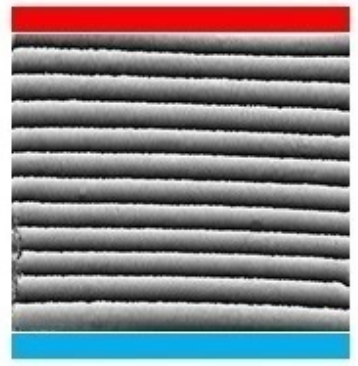

a)

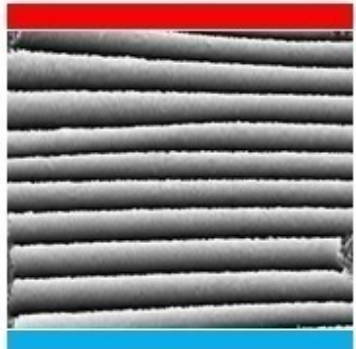

b)

$\nabla B^{2}$

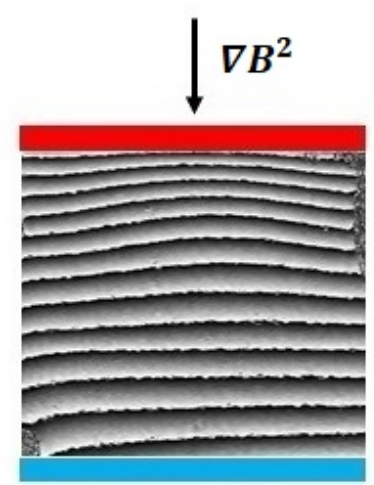

c)

Figure 5: MZI profiles for a $1.0 \mathrm{~mol} \mathrm{dm^{-3 }} \mathrm{Dy}^{3+}$ solution with configuration 1: a) $\Delta T=10$ ${ }^{\circ} \mathrm{C}, 30$ minutes; b) $\Delta T=10{ }^{\circ} \mathrm{C}$ with magnetic field applied from the bottom of the cell, 30 minutes; c) $\Delta T=10^{\circ} \mathrm{C}$ with magnetic field applied from the top of the cell, 30 minutes.

Changes in refractive indices were quantified for these three cases and are shown in Figure 6. When no magnetic field was applied, it was possible to observe an increase of refractive index in the bottom of the cell due to the cooling of the solution, and a decrease in the top due to the heating. When the magnetic field was applied from the bottom of the cell, the refractive index remained similar to the initial case. However, when the magnetic field was applied from the top, the magnetomigration of $\mathrm{Dy}^{3+}$ ions produced a small increase of refractive index towards the upper part of the cell. 


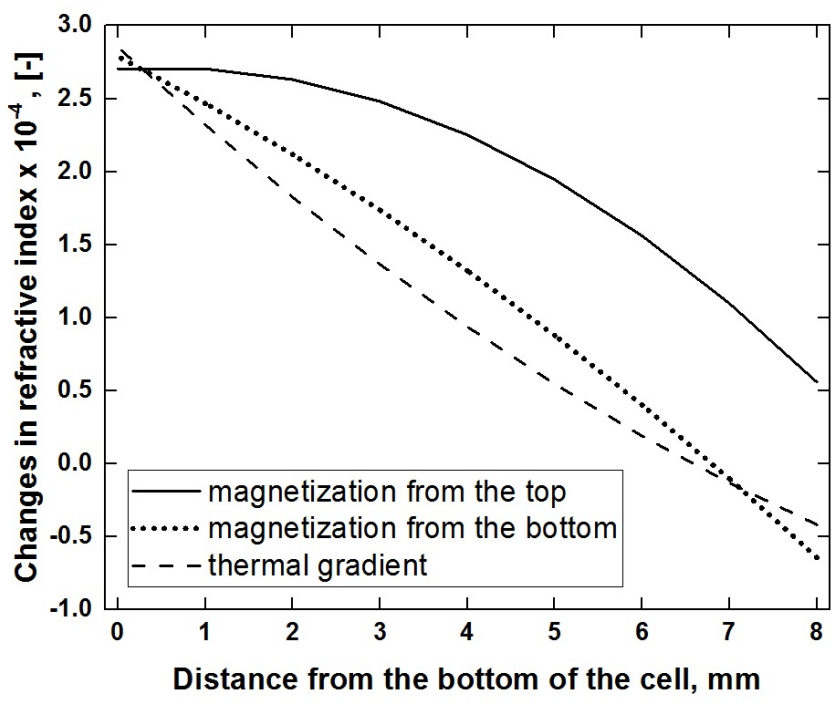

Figure 6: Refractive index change in a $1.0 \mathrm{~mol} \mathrm{dm}{ }^{-3} \mathrm{Dy}^{3+}$ solution due to: 30 minutes of $\Delta T=10{ }^{\circ} \mathrm{C}$ (dashed line); 30 minutes of magnetization applied from the bottom of the cell with $\Delta T=10{ }^{\circ} \mathrm{C}$ (dotted line); and 30 minutes of magnetization applied from the top of the cell with $\Delta T=10^{\circ} \mathrm{C}$ (black line).

Figure 7 shows the MZI profiles of experiments performed with the magnetic field applied from the left side of the cell. Here an enhanced magnetomigration was observed due to the higher non-collinearity of $\nabla B^{2}$ and $\nabla \chi$.

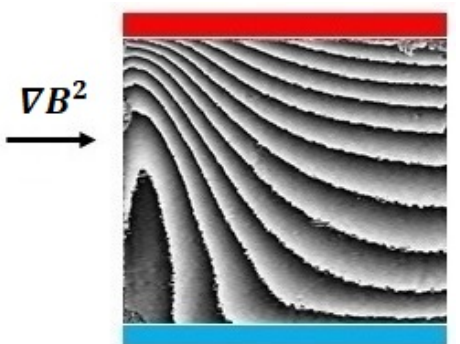

Figure 7: MZI profiles for a $1.0 \mathrm{~mol} \mathrm{dm}{ }^{-3} \mathrm{Dy}^{3+}$ solution with configuration $1, \Delta T=10^{\circ} \mathrm{C}$ with magnetic field applied from the left side of the cell.

Similarly to experiments described by Noddack et al., ${ }^{10-12}$ the configuration 2 of the heating/cooling device was used with the magnetic field perpendicular to the $\nabla \chi$ generated from the sides of the cell. In the Noddack's work, the magnetic field was applied near the bottom of the cell and fractions of different magnetic susceptibilities were collected after a certain period of magnetization uniformly over the cell height. Here, in order to improve 
qualitative observations of the magnetization and demagnetization processes, the magnetic field was applied from the top of the cell. Figure $8 \mathrm{a}$ shows the profile of a $1.0 \mathrm{~mol} \mathrm{dm}^{-3}$ $\mathrm{Dy}^{3+}$ solution after 30 minutes of magnetization with no thermal gradient applied. In this case, the system was initially homogeneous and the magnetization produced no detectable changes in the solution. Despite the presence of the magnet, the refractive index remained constant along the solution, within some small noise level. When a thermal gradient of 10 ${ }^{\circ} \mathrm{C}$ was applied alone, a convective fluid flow was developed (Figure 8b). In Figure 8c it is possible to observe the change in the fluid flow when the magnetic field was added to the experiment. The stratification of the solution became denser in the top of the cell due to the magnetomigration of the $\mathrm{Dy}^{3+}$ solution towards the region of the higher magnetic field. This enrichment becomes clear during the demagnitization step, the enriched $\mathrm{Dy}^{3+}$ solution convected back to the bulk in a mushroom-shaped downward plume (Figure 8d). 


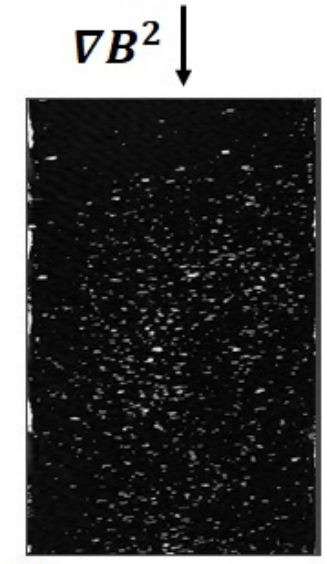

a)

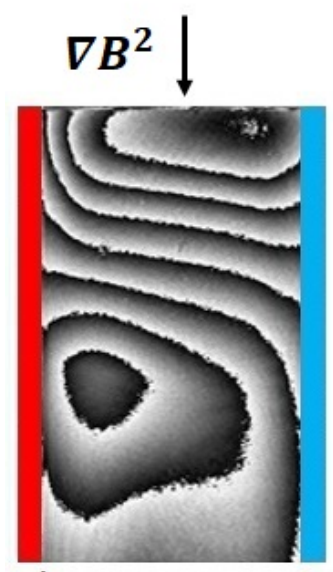

c)

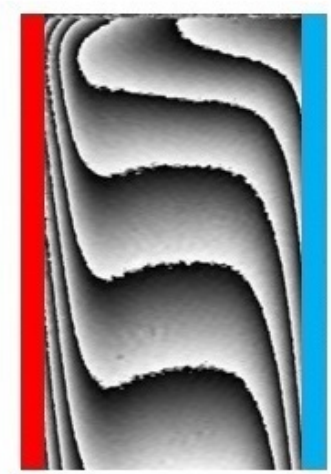

b)

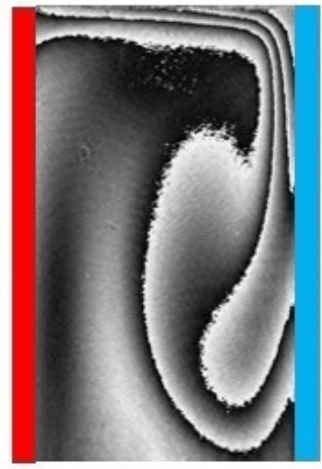

d)

Figure 8: MZI profiles for a $1.0 \mathrm{~mol} \mathrm{dm}^{-3} \mathrm{Dy}^{3+}$ solution using configuration 2 of the heating/cooling device: a) $\Delta T=0$, magnetization for 30 minutes; b) $\Delta T=10{ }^{\circ} \mathrm{C}, 30$ minutes without magnetization; c) $\Delta T=10{ }^{\circ} \mathrm{C}$, magnetization for 30 minutes; d) demagnetization: immediately after the magnet was removed.

\section{Magnetomigration with an evaporation-based gradient}

In our previous work, consistent magnetomigration in $\mathrm{Dy}^{3+}$ solution was obtained when magnetic susceptibility gradients were introduced by evaporation of the solvent. ${ }^{8}$ The $\nabla \chi$ created by the local densification of the solution activated the process of magnetomigration. However, the magnetomigration induced in the top of the cell at the liquid-air interface eventually led to Rayleigh-Bénard instabilities, causing plumes of denser solution to sink. A configuration with the magnet at the bottom of the cell was used here in order to avoid these 
instabilities and promote a synergic effect of magnetomigration and the local densification due to evaporation (Figure 3). MZI profiles for the evaporation and magnetomigration with evaporation for the strongly paramagnetic $\mathrm{Dy}^{3+}$ ions and weakly diamagnetic $\mathrm{Y}^{3+}$ ions are shown in Figure 9. Evaporation of solvent caused similar stratified profile in the bottom of the cell for both paramagnetic and diamagnetic species (Figure 9a). When the magnetic field was applied, the concentration gradient $\nabla c$ created by evaporation and thus the $\nabla \chi$ lead to magnetomigration of the $\mathrm{Dy}^{3+}$ solution. The attraction of paramagnetic $\mathrm{Dy}^{3+}$ solution to the higher magnetic field enlarged the stratified concentration profile at the bottom of the cell. The opposite effect would be expected for experiments with the diamagnetic $\mathrm{Y}^{3+}$ ions. However, no clear effect was detected due to the very weak form of magnetism induced when a diamagnetic compound is exposed to an external magnetic field.
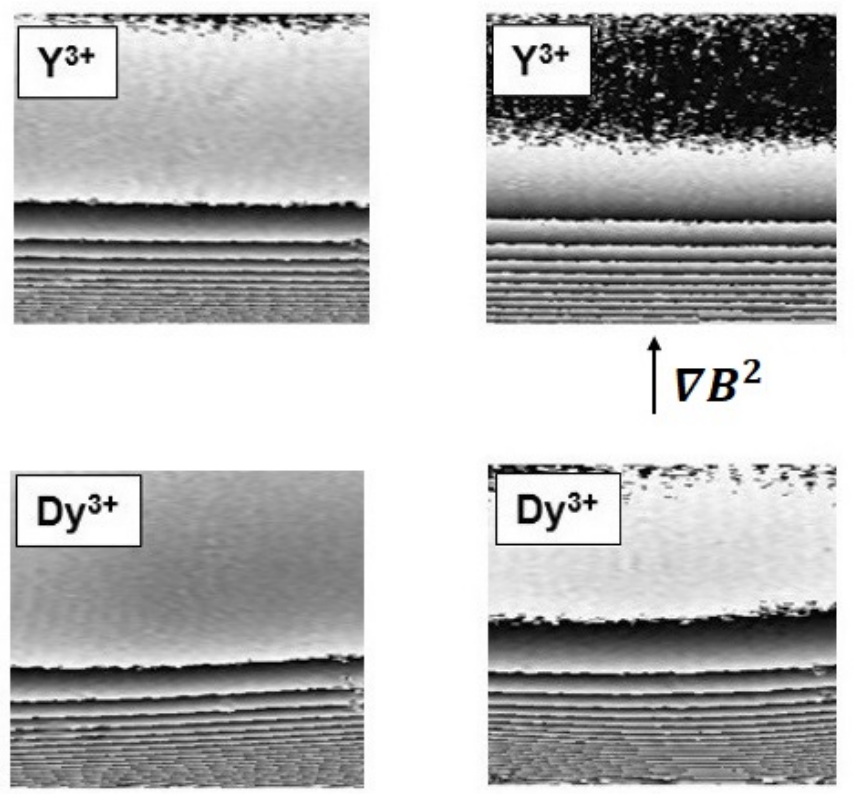

a)

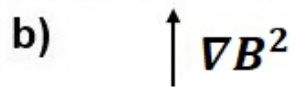

Figure 9: MZI profiles for a $1.0 \mathrm{~mol} \mathrm{dm}{ }^{-3}$ solutions of $\mathrm{Dy}^{3+}$ and $\mathrm{Y}^{3+}$ for 30 minutes of: a) evaporation and b) magnetomigration with evaporation-based gradient.

Figure 10 compares the change in refractive index along a $\mathrm{Dy}^{3+}$ solution during evaporation and magnetomigration with an evaporation-based gradient. 


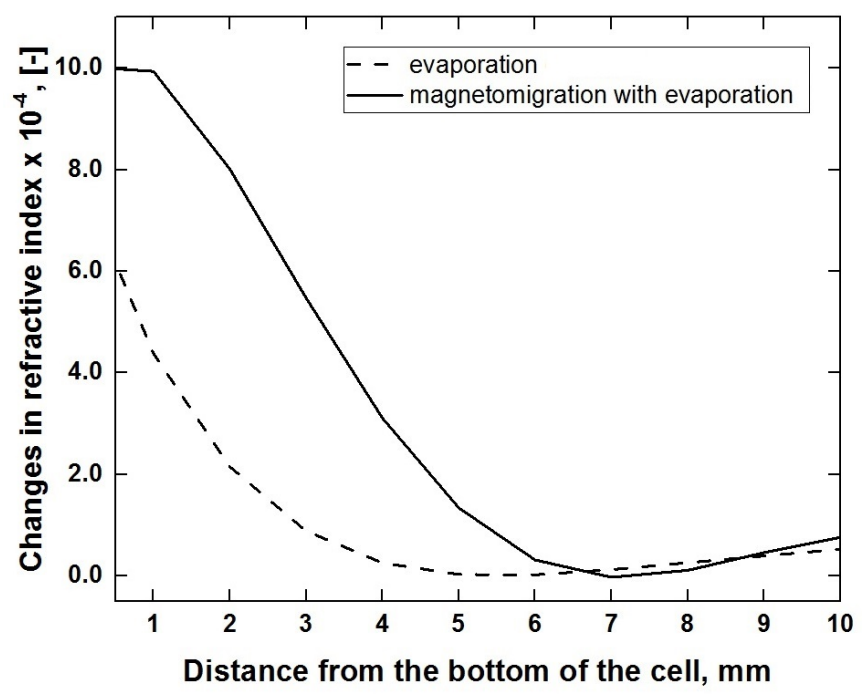

Figure 10: Refractive index change in a $1.0 \mathrm{~mol} \mathrm{dm}^{-3} \mathrm{Dy}^{3+}$ solution during 30 minutes of evaporation (dashed line) and magnetomigration with evaporation-based gradient (black line).

Disregarding other contributions that can affect the refractive index of a solution, concentration values were estimated based on the obtained refractive indices using the calibration curve shown in Figure S4 in the Supporting Information. The ionic enrichment due to evaporation and magnetomigration with evaporation were calculated as: $\Delta c=\frac{c_{\exp }-c_{\text {in }}}{c_{\text {in }}} \times 100$ [\%] (where $c_{\text {in }}$ is the initial concentration of the solution and $c_{\exp }$ is the concentration measured after 30 minutes of experiment). The highest changes in the refractive index values, in the region close to the bottom of the cell, were used to compare the $\mathrm{Dy}^{3+}$ enrichment due to evaporation (dashed line in Figure 10) and magnetomigration with evaporation (black line in Figure 10). The $\mathrm{Dy}^{3+}$ enrichment due to evaporation was calculated as $1.6 \%$ and the enrichment due to magnetomigration with evaporation $2.7 \%$, with the contribution of the magnetic effect alone can be estimated $\approx 1 \%$. To compare the magnetomigration of paramagnetic solutions with different magnetic susceptibilities, experiments were also conducted with $1.0 \mathrm{~mol} \mathrm{dm}{ }^{-3}$ solutions of the paramagnetic $\mathrm{Nd}^{3+}$ and $\mathrm{Gd}^{3+}$. The electron configuration of the $\mathrm{RE}^{3+}$ ions, the magnetic susceptibility of corresponding $\mathrm{RE}\left(\mathrm{NO}_{3}\right)_{3}$ solution and the ionic enrichment due to magnetomigration with 
evaporation are displayed in Table 1 . In the series $\mathrm{Nd}^{3+}, \mathrm{Gd}^{3+}, \mathrm{Dy}^{3+}$, the values of ionic enrichment increase proportional to the magnetic susceptibility of the ions. The influence of the magnetic anisotropy on the magnetomigration has been neglected in this study. First of all, the $\mathrm{Gd}^{3+}$ ion shows magnetomigration although it is magnetically isotropic (the magnetic anisotropy is equal to zero). Secondly, Binnemans et al. have shown that for coordination polyhedra typically found for hydrated lanthanide ions, a very small magnetic anisotropy is predicted. ${ }^{35}$

Table 1: Electron configuration of $\mathrm{RE}^{3+}$ ions, measured $\chi_{\text {sol }}$ of the $1.0 \mathrm{~mol} \mathrm{dm}{ }^{-3} \mathrm{RE}\left(\mathrm{NO}_{3}\right)_{3}$ solutions and ionic enrichment obtained due to magnetomigration with an evaporation-based gradient.

\begin{tabular}{cccc}
\hline Ion & Electron configuration & $\chi[-]$ & $\Delta c[\%]$ \\
\hline $\mathrm{Nd}^{3+}$ & {$[\mathrm{Xe}] 4 \mathrm{f}^{3}$} & $+3.58 \times 10^{-5}$ & 1.1 \\
$\mathrm{Gd}^{3+}$ & {$[\mathrm{Xe}] 4 \mathrm{f}^{7}$} & $+21.79 \times 10^{-5}$ & 2.0 \\
$\mathrm{Dy}^{3+}$ & {$[\mathrm{Xe}] 4 \mathrm{f}^{9}$} & $+39.40 \times 10^{-5}$ & 2.7 \\
\hline
\end{tabular}

Even though $\nabla \chi$ and $\nabla B^{2}$ in experiments with evaporation-based gradients are not aligned in the highest degree of non-collinearity, clear magnetomigration was observed for all tested paramagnetic ions. The $\mathrm{Dy}^{3+}$ enrichment due solely to magnetomigration was one order of magnitude higher when compared to the one obtained using $\Delta T$ as source of $\nabla \chi$, which was $\approx 0.10 \%$, as calculated from the refractive index profile shown in Figure 6 , black line. This can be explained once $\nabla \chi$ 's generated by evaporation are larger and more localized than the gradients created by $\Delta T$.

\section{Effect of a non-static magnetic field on the magnetomigration}

When the magnet was equipped with a magnetic field concentrator and moved along the bottom of the cell, layers of enriched $\mathrm{Dy}^{3+}$ solution followed the magnetic field. The 
stratified solution moved from the center of the cell (Figure 11a), to the left wall (Figure 11b) and to the right wall (Figure 11c). These experiments allow to more clearly separate the effect of the magnetic field on the initial stratification of the solution due to evaporation.

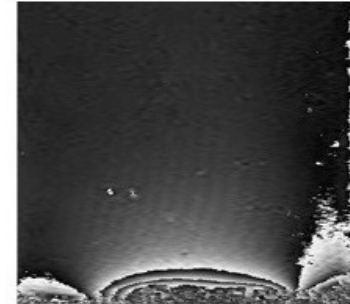

a)

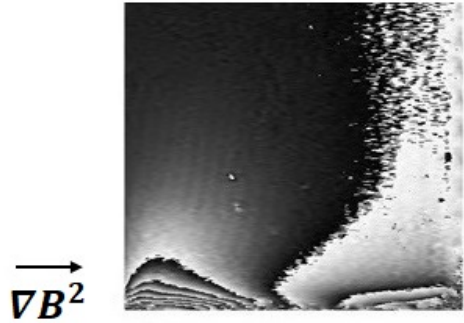

b)

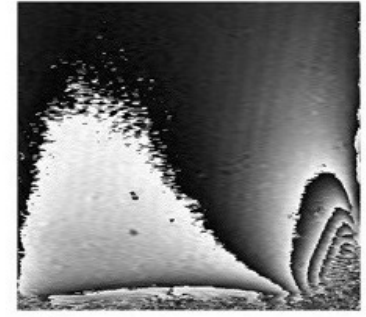

c)

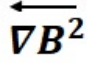

$\overleftarrow{\nabla B^{2}}$

Figure 11: MZI profiles for a $1.0 \mathrm{~mol} \mathrm{dm}^{-3} \mathrm{Dy}^{3+}$ solution and magnetomigration with evaporation-based gradient. The magnet was moved at $5 \mathrm{~mm} \mathrm{~s}^{-1}$ from: a) the center of the cell; b) to the left side of the cell and c) to the right side of the cell.

\section{Magnetomigration experiments with removal of thermal and evaporation-based gradients}

The effect of the removal of the thermal and evaporation-based gradient during magnetomigration was tested using a $\mathrm{Dy}^{3+}$ solution. Figure 12 shows the MZI profiles obtained in an experiment where the thermal gradient was cancelled after 5 minutes of magnetization. In the beginning of the experiment, the magnetomigration of the $\mathrm{Dy}^{3+}$ solution was observed in the stratified region at the top of the cell, close to the magnet (Figure 12a). However, when $\Delta T$ was reduced to zero, the magnetomigration effect was gradually lost (Figure 12b). After 10 minutes the solution reached a quasi-homogeneous state despite the presence of the magnetic field (Figure 12c). 


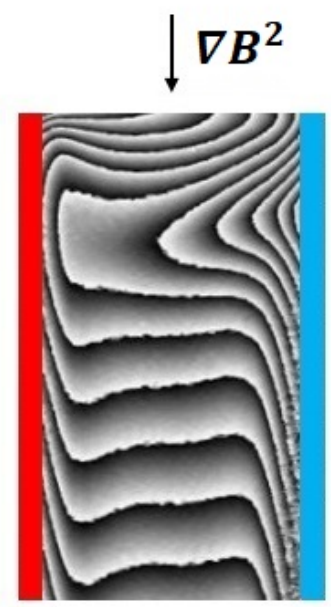

a)

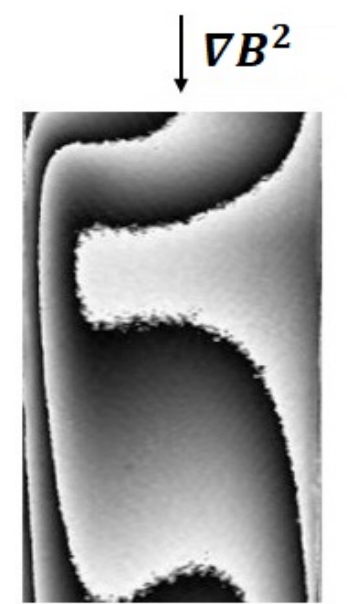

b)

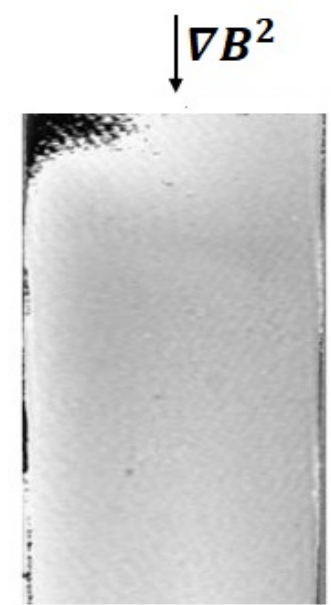

c)

Figure 12: MZI profiles for a $1.0 \mathrm{~mol} \mathrm{dm}{ }^{-3} \mathrm{Dy}^{3+}$ solution, magnetomigration experiment with removal of the thermal gradient: a) $\Delta T=10{ }^{\circ} \mathrm{C}, 5$ minutes of magnetization; b) gradual loss of magnetic effect with $\Delta T=0$; and c) loss of magnetic effect when $\Delta T=0$ for 10 minutes.

A similar effect was observed for experiments with an evaporation-based gradient. When the evaporation was stopped by closing the space between the PTFE membrane and the magnet after 5 minutes of magnetization, the stratified profile due to the enrichment of $\mathrm{Dy}^{3+}$ ions disappeared within 10 minutes (Figure 13). This demonstrates that the magnetic force is deactivated in the absence of $\nabla \chi$. 


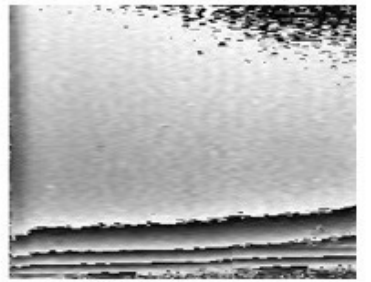

a)

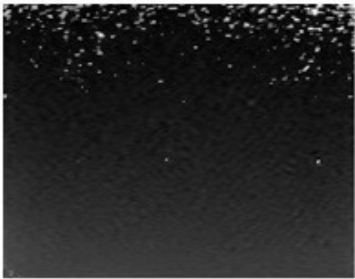

b) $\uparrow \nabla B^{2}$

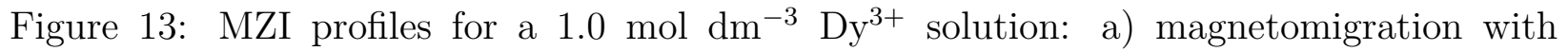
evaporation-based gradient, 5 minutes; and b) loss of magnetic effect with blocked evaporation, 10 minutes.

\section{Conclusions}

Two different methods to induce the magnetomigration of rare-earth ions in solution were demonstrated. Both methods were based on the activation of the rotational component of the magnetic field gradient force. The use of a heating/cooling device allowed to generate gradients of magnetic susceptibility in the solution due to temperature dependence of $\chi_{\mathrm{m}}$, which in turn induced the magnetomigration. During experiments with $\Delta T$, layers of enriched $\mathrm{Dy}^{3+}$ solution were formed in the region of the strongest magnetic field when the magnetic field was applied from the top and the side of the cell. Stable zones of ionic enrichment were formed during magnetomigration experiments with evaporation-based gradients for all tested paramagnetic $\mathrm{RE}$ solutions: $\mathrm{Nd}^{3+}, \mathrm{Gd}^{3+}$ and $\mathrm{Dy}^{3+}$. It was shown that the degree of ionic enrichment is proportional to the magnetic susceptibility of the REs. A maximum enrichment of $1 \%$ was obtained for the strongly paramagnetic $\mathrm{Dy}^{3+}$ ions after 30 minutes of magnetization. Additionally, when the thermal gradient and the evaporation-based gradient were removed during the experiments by means of stopping the $\Delta T$ or blocking the evaporation, the magnetization effect gradually disappeared. It was previously assumed that the presence of magnetic susceptibility gradients contributed to the magnetomigration as a trigger ${ }^{8}$. Results presented here indicate that not only a constant input of $\nabla \chi$ is mandatory for the magnetomigration 
effect, but also the non-collinear alignment of $\nabla \chi$ and $\nabla B$. These findings represent an important step towards the development of magnetic separation methods for RE ions.

\section{Associated Content}

\section{Supporting Information}

Experimental description and detailed setup of the Mach-Zehnder interferometry. Characterization of a $1.0 \mathrm{~mol} \mathrm{dm}{ }^{-3} \mathrm{Dy}^{3+}$ solution (refractive index with concentration and

temperature; magnetic susceptibility with temperature; and evaporation rate) and measurement of the magnetic field flux of the permanent magnet used. Simulation of the temperature dependence of magnetic susceptibility with $\Delta T$. Calibration of the Peltier elements. Videos of the magnetomigration of $\mathrm{Dy}^{3+}$ ions with an evaporation-based gradient. Video of the magnetomigration experiment with the removal of $\Delta T$. This material is available free of charge via the Internet at http://pubs.acs.org.

\section{Author Information}

*Email - Isadora.Reisrodrigues@kuleuven.be

*ORCID iD - https://orcid.org/0000-0002-3121-973X

\section{Authors Contributions}

The manuscript was written through contributions of all authors. All authors have given approval to the final version of the manuscript.

\section{Note}

The authors declare no competing financial interest. 


\section{Acknowledgement}

The authors acknowledge funding from KU Leuven (DBOF grants to Isadora R. Rodrigues and Liubov Lukina), FWO Flanders (project G082716N), EVAPORATION and HEAT TRANSFER projects funded by the European Space Agency and the Belgian Science Policy Office PRODEX Programme, as well as the FRS-FNRS. The authors also thank Joop van Deursen of the Department of Materials Engineering at KU Leuven for the design of the heating/cooling device and the magnetic field concentrator.

\section{References}

(1) Binnemans, K.; Jones, P. T.; Blanpain, B.; Van Gerven, T.; Pontikes, Y. Towards ZeroWaste Valorisation of Rare-Earth-Containing Industrial Process Residues: A Critical Review. J. Clean Prod. 2015, 99, 17-38.

(2) Cotton, S. Lanthanides and actinides; Wiley: Chichester, 2006.

(3) Rodrigues, I.; Lukina, L.; Dehaeck, S.; Colinet, P.; Binnemans, K.; Fransaer, J. Magnetophoretic Sprinting: A Study on the Magnetic Properties of Aqueous Lanthanide Solutions. J. Phys. Chem. C 2018, 41, 23675-23682.

(4) Oberteuffer, J. Magnetic Separation: A Review of Principles, Devices, and Applications. IEEE Trans. Magn. 1974, 10, 223-238.

(5) Kelland, D.; Kolm, H.; deLatour, C.; Maxwell, E.; Oberteuffer, J. Superconducting Machines and Devices: Large Systems Applications; Springer US: Boston, 1974.

(6) Takayasu, M.; Gerber, R.; Friedlaender, F. Magnetic Separation of Submicron Particles. IEEE Trans. Magn. 1983, 19, 2112-2114.

(7) Coey, J. Magnetism and Magnetic Materials; Cambridge University Press: Cambridge, 2010. 
(8) Rodrigues, I.; Lukina, L.; Dehaeck, S.; Colinet, P.; Binnemans, K.; Fransaer, J. Magnetomigration of Rare-Earth Ions Triggered by Concentration Gradients. J. Phys. Chem. Lett. 2017, 8, 5301-5305.

(9) Dunne, P.; Soucaille, R.; Ackland, K.; Coey, J. Structuring of Electrodeposits with Permanent Magnet Arrays. Magnetohydrodynamics 2012, 48, 331-342.

(10) Noddack, W.; Wicht, E. Eine Neue Methode zur Trennung der Seltenen Erden. Ber. Bunsenges. Phys. Chem. 1952, 56, 893-895.

(11) Noddack, I.; Wicht, E. Trennung der Seltenen Erden im Inhomogenen Magnetfeld. Chem. Techn. 1955, r, 3-5.

(12) Noddack, W.; Noddack, I.; Wicht, E. Zur Trennung der Seltenen Erden im Inhomogenen Magnetfeld. Ber. Bunsenges. Phys. Chem. 1958, 62, 77-85.

(13) Mukherjee, H.; Datta, S. K. Magneto-Paper Electrophoresis in the Separation of Inorganic Ions. Microchim. Acta 1983, 79, 431-436.

(14) Franczak, A.; Binnemans, K.; Fransaer, J. Magnetomigration of Rare-Earth Ions in Inhomogeneous Magnetic Fields. Phys. Chem. Chem. Phys. 2016, 18, 27342-27350.

(15) Kolzcyk, K.; Wojnicki, M.; et al., D. K. Separation of $\mathrm{Ho}^{3+}$ in Static Magnetic Field. Arch. Metall. Mater. 2017, 61, 1919-1924.

(16) Fujiwara, M.; Kodoi, D.; Duan, W.; Tanimoto, Y. Separation of Transition Metal Ions in an Inhomogeneous Magnetic Field. J. Phys. Chem. B 2001, 105, 3343-3345.

(17) Fujiwara, M.; Chie, K.; Sawai, J.; Shimizu, D.; Tanimoto, Y. On the Movement of Paramagnetic Ions in an Inhomogeneous Magnetic Field. J. Phys. Chem. B 2004, 108, $3531-3534$.

(18) Fujiwara, M.; Mitsuda, K.; Tanimoto, Y. Movement and Diffusion of Paramagnetic Ions in a Magnetic Field. J. Phys. Chem. B 2006, 110, 13965-13969. 
(19) Chie, K.; Fujiwara, M.; Fujiwara, Y.; Tanimoto, Y. Magnetic Separation of Metal Ions. J. Phys. Chem. B 2003, 10\%, 14374-14377.

(20) Yang, X.; Tschulik, K.; Uhlemann, M.; Odenbach, S.; Eckert, K. Enrichment of Paramagnetic Ions from Homogeneous Solutions in Inhomogeneous Magnetic Fields. J. Phys. Chem. Lett. 2012, 3, 3559-3564.

(21) Yang, X.; Tschulik, K.; Uhlemann, M.; Odenbach, S.; Eckert, K. Magnetic Separation of Paramagnetic Ions From Initially Homogeneous Solutions. IEEE Trans. Magn. 2014, $50,4600804-4600808$.

(22) Pulko, B.; Yang, X.; Lei, Z.; Odenbach, S.; Eckert, K. Magnetic Separation of Dy(III) Ions from Homogeneous Aqueous Solutions. Appl. Phys. Lett. 2014, 105, 232407232411.

(23) Lei, Z.; Fritzsche, B.; Eckert, K. Evaporation-Assisted Magnetic Separation of RareEarth Ions in Aqueous Solutions. J. Phys. Chem. C 2017, 121, 24576-24587.

(24) Coey, J. M. D.; Rhen, F. M. F.; Dunne, P.; McMurry, S. The Magnetic Concentration Gradient Force-Is it Real? J. Solid State Electrochem. 2007, 11, 711-717.

(25) Hinds, G.; Coey, J.; Lyons, M. Influence of Magnetic Forces on Electrochemical Mass Transport. Electrochem. Commun. 2001, 3, 215-218.

(26) Pamme, N. Magnetism and Microfluidics. Lab. Chip 2006, 6, 24-38.

(27) Monzon, L. M.; Coey, J. Magnetic Fields in Electrochemistry: The Kelvin Force. A Mini-Review. Electrochem. Commun. 2014, 42, 42-45.

(28) Mutschke, G.; Tschulik, K.; Weier, T.; Uhlemann, M.; Bund, A.; Fröhlich, J. On the Action of Magnetic Gradient Forces in Micro-Structured Copper Deposition. Electrochim. Acta 2010, 55, 9060-9066. 
(29) Uhlemann, M.; Tschulik, K.; Gebert, A.; Mutschke, G.; Fröhlich, J.; Bund, A.; Yang, X.; Eckert, K. Structured Electrodeposition in Magnetic Gradient Fields. Eur. Phys. J. Spec. Tор. 2013, 220, 287-302.

(30) Tschulik, K.; Cierpka, C.; Mutschke, G.; Gebert, A.; Schultz, L.; Uhlemann, M. Clarifying the Mechanism of Reverse Structuring During Electrodeposition in Magnetic Gradient Fields. Anal. Chem. 2012, 84, 2328-2334.

(31) Rahman, M.; Saghir, M. Thermodiffusion or Soret Effect: Historical Review. Int. J. Heat Mass Transfer 2014, 73, 693-705.

(32) Kreis, T. Digital Holographic Interference-Phase Measurement Using the FourierTransform Method. J. Opt. Soc. Am. A 1986, 847-855.

(33) Dehaeck, S.; Wylock, C.; Colinet, P. Evaporating Cocktails. Phys. Fluids 2009, 21, 091108.

(34) Aogaki, R.; Ito, E.; Ogata, M. A New Flow-Type Cell by the Application of Magnetic Microfluidic Chip. J. Solid State Electrochem. 2007, 11, 757-762.

(35) Mironov, V. S.; Galyametdinov, Y. G.; Ceulemans, A.; Görller-Walrand, C.; Binnemans, K. Room-Temperature Magnetic Anisotropy of Lanthanide Complexes: A Model Study for Various Coordination Polyhedra. J. Chem. Phys. 2002, 116, 46734685. 


\section{Graphical TOC Entry}

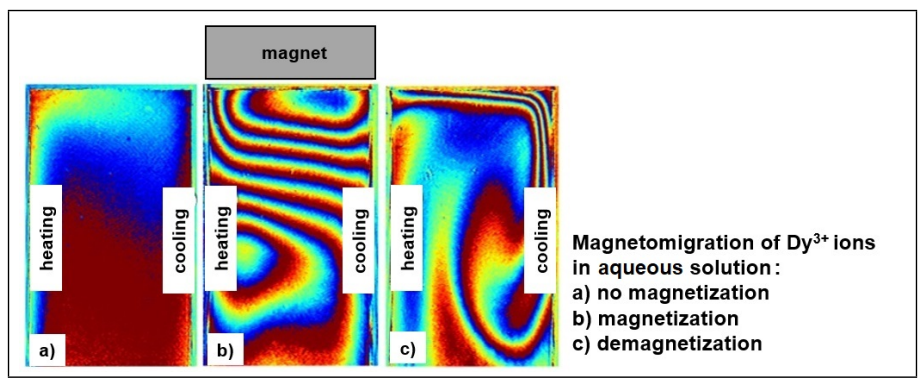

